\title{
Effects of Topical Ozone Application on Outcomes after Accelerated Corneal Collagen Cross-linking: An Experimental Study
}

\author{
Aysun Sanal Dogan, MD, FEBO, FICO'; Canan Gurda, MD'; Sinan Caliskan, MD, FEBO'; Evrim Onder, MD $^{2}$ \\ Figen Kaymaz, MD ${ }^{3}$; Elif Bilgic, MD $^{3}$
}

\author{
${ }^{1}$ Department of Ophthalmology, Saglik Bilimleri University, Diskapi Yildirim Beyazit Training and Research Hospital, Ankara, \\ Turkey \\ ${ }^{2}$ Department of Pathology, Saglik Bilimleri University, Diskapi Yildirim Beyazit Training and Research Hospital, Ankara, Turkey \\ ${ }^{3}$ Department of Histology and Embryology, Hacettepe University, School of Medicine, Ankara, Turkey
}

ORCID:

Aysun Sanal Dogan: https://orcid.org/0000-0002-7401-8903

\begin{abstract}
Purpose: Ozone is a trioxygen molecule that spontaneously degrades into oxygen and oxygen free radicals. This study was designed to assess the effects of topical ozone application on outcomes after corneal collagen cross-linking (CXL).

Methods: Enucleated fresh cadaver yearling sheep eyes $(n=28)$ were divided into five groups: control (C, $n=6)$, sham (S, $n=6$ ), ozone only $(\mathrm{Z}, n=6)$, CXL only $(\mathrm{X}, n=5)$, and Ozone $+\mathrm{CXL}(\mathrm{ZX}, n$ $=5$ ). In all groups, except $C$, the epithelial layer was removed. In group Z, $20 \mu \mathrm{g} / \mathrm{mL}$ liquid ozone was topically applied. In group X, CXL was performed in the accelerated pulse mode. In group $Z X$, both $C X L$ and ozone were applied. Post-interventional oxygen levels were determined and corneal confocal microscopy and optical coherence tomography were performed. Corneas were evaluated using light and electron microscopy.

Results: Pre-interventional central corneal thickness (CCT) was highest in the control group and considerably similar in the remaining groups $(P=0.006)$. Pre- and post-interventional CCT were significantly different in the ozonated groups $(Z$ and $Z X)(P=0.028 ; P=0.043)$. Demarcation line depths were similar in groups $Z, X$, and $Z X(P=0.343)$. Increased stromal tissue reflectivity was observed in groups $Z, X$, and $Z X$. Oxygen levels were higher in the ozonated groups ( $Z$ and $Z X)(P=0.006)$, and caspase activity was higher in the CXL groups (X and $Z X)(P=0.028)$ as compared to the other groups. Group ZX showed tighter, more regular, and parallel fibrils. Conclusion: Ozone increases corneal stromal oxygenation which can probably augment the effect of CXL. Future studies should investigate the safety and feasibility of ozone application during CXL.
\end{abstract}

Keywords: Ozone; Corneal Collagen Cross-linking; Corneal Confocal Microscopy; Corneal Oxygen; Experimental

J Ophthalmic Vis Res 2020; 15 (3): 289-298

Correspondence to:

Aysun Sanal Dogan, MD, FEBO, FICO. Diskapi Yildirim Beyazit Egitim ve Arastirma Hastanesi, Sehit Omer Halisdemir Cad. Diskapi 06130, Ankara, Turkey. E-mail: asanaldogan@gmail.com

Received: 02-10-2019 Accepted: 03-04-2020

\section{Access this article online}

Website:

https://knepublishing.com/index.php/JOVR

DOI:

10.18502/jovr.v15i3.7447

\section{INTRODUCTION}

The cornea contains outer epithelial cell layer, middle stromal layer, and inner endothelial

This is an open access journal, and articles are distributed under the terms of the Creative Commons Attribution-NonCommercial-ShareAlike 4.0 License, which allows others to remix, tweak, and build upon the work non-commercially, as long as appropriate credit is given and the new creations are licensed under the identical terms.

How to cite this article: Dogan AS, Gurda C, Caliskan S, Onder E, Kaymaz F, Bilgic E. Effects of Topical Ozone Application on Outcomes after Accelerated Corneal Collagen Cross-linking: An Experimental Study. J Ophthalmic Vis

Res 2020;15:289-298. 
cell layer. The stromal layer comprises the major volume of the tissue and is composed of keratocytes and extracellular matrix (ECM) including collagen lamella. The shape and structure of the corneal stroma are primarily responsible for the transparency, strength, and contour of the cornea. ${ }^{[1]}$

Keratoconus $(\mathrm{KC})$ is a non-inflammatory ectatic disorder of the cornea, characterized by progressively bulging and thinning in the central or paracentral area of the cornea. ${ }^{[2]}$ Ultrastructural changes of stromal matrix include increased corneal stromal protein degradation, decreased collagen lamella density, and disorganization of the stromal matrix, resulting in biochemical instability and weakening. ${ }^{[3]}$ Contemporary treatment has been undertaken with the advent of corneal collagen cross-linking (CXL), demonstrating promising results in terms of halting disease progression or even, to a limited extent, reversing the course of KC. ${ }^{[4]}$

The application of riboflavin and ultraviolet A during the $C X L$ procedure results in the formation of oxygen radicals which crosslink in the adjacent collagen fibrils. This cross-linking increases the biomechanical strength by modifying the organization of the corneal lamellar structure, the diameter of the corneal stromal collagen, and the spacing between fibrils and proteoglycans. ${ }^{[5]}$ It has been shown that tissue oxygen is the key element for this reaction to occur. ${ }^{[6]}$

Within the last five years, a growing number of studies have been performed to facilitate the procedure without decreasing the efficacy. These studies have focused on the four basic variables of this procedure.

1 Delivery of riboflavin to cornea: Chemical de-epithelization, the "epi-on" (without deepithelization) and intraoperative contact lens using techniques to decrease the operative complications and postoperative pain, and "iontophoresis technique" to increase efficacy have been described. ${ }^{[7,8]}$

2 Photosensitizing agent: Reportedly, studies have evaluated dosage modification and duration of riboflavin application; additionally, alternatives to this original photosensitizing agent have been investigated. ${ }^{[9]}$

3 Duration of exposure to UVA: The reaction is initiated by UVA. In 2014, "accelerated CXL" was described and aimed to shorten the duration by increasing the UVA irradiance, without changing the total energy. ${ }^{[10]}$

4 Oxygen: The photochemical reaction requires oxygen. Following the realization that environmental oxygen is rapidly depleted within seconds and rises to normal limits after $3 \mathrm{~min}$, it was proposed that the "pulse CXL" method allows tissue reoxygenation. ${ }^{[6,11,12]}$

The effect of cross-linking on collagen was first investigated using the crystalline lens. ${ }^{[13]}$ The lens and lenticular collagen were the first subjects of these studies, which demonstrated the interaction among singlet oxygen, ozone, and applied riboflavin that resulted in free radicalinduced cross-linking of the lenticular fibrils. ${ }^{[14-16]}$ These aforementioned studies put forward the possible use of ozone in CXL.

Ozone is an unstable trioxygen molecule. Its breakdown to oxygen gives rise to oxygen free radicals, which are highly reactive and powerful oxidizing agents. ${ }^{[17]}$ As a result, it serves as an oxygen supply. Therefore, by itself or in conjunction with riboflavin and UVA, it has the potential to augment the cross-linking effect. We hypothesized that ozone can be used as an adjuvant to the cross-linking reaction, as an oxygen generator, or as a cross-linking agent in the cornea. This study was designed to assess the effect of topical ozone application on the outcomes of the CXL procedure.

\section{METHODS}

\section{Study Design}

Enucleated fresh cadaver yearling sheep eyes ( $n$ $=28$ ) were obtained from a local slaughterhouse and the full experimental procedure was performed within 12 hours. The eyes were divided into five groups: control (C, $n=6)$, sham (S, $n=6)$, ozone only (Z, $n=6)$, CXL only (X, $n=5)$, and Ozone $+\mathrm{CXL}$ $(Z X, n=5)$.

During the experiment, the eyes were handled from the equator using gauze sponges. Group $\mathrm{C}$ was not touched or handled, and the epithelial layer was mechanically removed for all other groups. In group $S$, only the epithelial layer was mechanically removed. In group Z, $20 \mu \mathrm{g} / \mathrm{mL}$ ozonated water was topically applied to the deepithelized cornea. In group X, CXL was performed using a total energy of $5,4 \mathrm{~J} / \mathrm{cm}^{2}$ in the accelerated 
pulse mode. ${ }^{[11]}$ In group ZX, ozone was first applied, followed by CXL treatment. Pre- and postinterventional anterior segment optical coherence tomography (AS-OCT) and corneal confocal microscopy (CCM) were performed. Immediate post-interventional corneal stromal oxygen levels were measured. After the procedure, each cornea was dissected from the globe and specimens were obtained to perform light and electron microscopic evaluations.

\section{Ozone Application}

Liquid ozone $(20 \mu \mathrm{m} / \mathrm{mL})$ was obtained using an ozone generator (Refresh, Refreshozon Medical, Ankara, Turkey) in distilled water in a separate room. Two milliliters of the ozonated water was applied to the cornea for 2 min using a silica glass apparatus, with a corneal $8 \mathrm{~mm}$ aperture.

\section{Cross-linking}

After removal of the central $8 \mathrm{~mm}$ portion of the epithelium using a No. 15 knife, 0.1\% riboflavin (vitamin B2) with hydroxypropyl methylcellulose (VibeXRapid, Avedro) was applied drop-wise to the cornea at 2 min intervals for $10 \mathrm{~min}$ to achieve corneal penetration. Anterior segment diffusion was controlled using handheld biomicroscope. A UVA (370 nm) generator designed for corneal cross-linking was used (KXL System, Avedro Inc., Waltham, MS, USA). First, guiding lights were set to focus the UVA onto the cornea perfectly. The parameters were set as power: $15 \mathrm{~mW} / \mathrm{cm}^{2}$, a total energy of $5.4 \mathrm{~J} / \mathrm{cm}^{2}$ for each eye, with a pulse of 1.5 -sec on and 1.5-sec off mode.

\section{Oxygen Measurement}

Post-interventional oxygen measurements were performed at room temperature $\left(21^{\circ} \mathrm{C}\right)$. The operator was masked to the groups. An oxygen sensor microprobe (PreSens, Regensburg, Germany), with a tapering end $<50 \mu \mathrm{m}$ in diameter, was placed from the epithelized cornea, passing $2 \mathrm{~mm}$ into the de-epithelized cornea, with a parallel angle at the half-thickness of the cornea under the biomicroscope. The mean of three consecutive measurements was used for statistical analyses.

\section{Anterior Segment Optical Coherence Tomography (AS-OCT)}

Corneas were imaged using optical coherence tomography (OCT, RTVue-XR, Optovue Inc., Fremont, CA). The non-contact anterior segment attachment lens (CAM-L), corneal line mode, and automated image analysis system were used. The sections were set to the central cornea. In particular, central corneal thickness (CCT) was measured in micrometers and the depth of demarcation lines, which are the results of different reflectivity, were investigated. The reflective line between the cross-linked and untreated areas was determined and marked.

\section{Corneal Confocal Microscopy (CCM)}

Laser scanning CCM (HRT III-RCM, Heidelberg Engineering, Dossenheim, Germany) was used to evaluate the cellular structures of the corneas. A transparent ophthalmic gel (Viscotears Ophthalmic Gel, Alcon) was filled into the confocal cap attached to the objective lens and on its external surface. The eyes were handled with a sponge from the equator, providing the central corneal touch to the cap. The captured images were analyzed by a clinician (ASD) experienced in CCM.

\section{Light Microscopy}

Caspase-3 staining was performed to detect keratocyte apoptosis. Paraffin-embedded tissue blocks were sectioned into $4-5 \mu \mathrm{m}$ thick slides. The slides were deparaffinized, rehydrated, and washed in phosphate-buffered saline (PBS). After treatment with $3 \%$ hydrogen peroxide in aqueous solution, the sections were blocked with PBS-6\% non-fat dry milk for 1 hour at room temperature. The slides were then incubated at $4{ }^{\circ} \mathrm{C}$ overnight with the primary antibody for cleaved caspase3 (CPP32) (Thermo Scientific). After washing with PBS, the slides were treated with a solution of diaminobenzidine (DAB). Finally, a counterstain with hematoxylin was performed and the slides were allowed to dry. The immunohistochemical $(\mathrm{IHC})$ evaluation was performed to determine the nuclei of stromal cells, and positivity was scored as percentages. Caspase activity was evaluated in stromal cells. In each section, the area with the highest density of nuclear positivity was selected 
and 100 cells were counted. The ratio of positively stained nuclei to 100 (the percentage value) was accepted as the caspase activity for each given case.

\section{Electron Microscopy}

Tissue samples were carefully dissected and $1 \mathrm{~mm}^{3}$-sized samples were fixed overnight in $2.5 \%$ glutaraldehyde in PBS. Next, the tissue samples were washed with PBS and fixed with $1 \%$ osmium tetroxide solution. After washing with PBS, the tissue samples were dehydrated with a graded alcohol series. An Araldite/Epon812 mixture (Cat no.: 13940, EMS, Hatfield, PA, USA) was used to embed the propylene-oxide-treated tissue. The tissue blocks obtained were maintained at $60^{\circ} \mathrm{C}$ for two days to complete polymerization. Thin sections were obtained using the Leica Ultracut R, and contrast double-stained using uranyl acetate and lead citrate (Leica EM AC20). Next, examinations were performed by using transmission electron microscope (TEM; JEM 1400, Jeol, Japan) with an attached digital CCD camera (Gatan Inc., Pleasanton, CA, USA). To compare the differences among the groups, we obtained four blocks from each group, and then examined four sections from each block. Finally, collagen fibril diameters and ECM distances from four non-overlapping areas were calculated at the magnification of $100 \mathrm{k}$.

\section{RESULTS}

Tissue oxygen levels were higher in the ozonated groups ( $Z$ and $Z X)$ than in the other groups $(P=$ 0.006, Kruskal-Wallis test). Pre-interventional CCT measures were higher in the control group (C) as compared to the other groups $(P=0.006)$; no significant difference, however, was found among the remaining groups in the baseline CCT. Postinterventional CCT differed significantly among the groups $(P=0.001)$. Compared to the baseline values, the post-interventional CCT decreased significantly in groups $Z$ and $Z X$ (Group Z: $P=0.028$, Group X: $P=0.768$, Group ZX: $P=0.043$; Table 1). Similar demarcation lines were observed in the interventional groups, although group ZX showed a generalized increase in corneal reflectivity (Figure 1).

CCM demonstrated corneal stromal hyperreflectivity (group $Z, X$, and $Z X$ ) in all interventional groups; however, the hyper-reflectivity was marked and present in all layers of the stroma in group $Z \mathbf{Z X}$ (Figure 2).

The cross-linked groups (groups $X$ and $Z X$ ) showed higher caspase activity, with significant differences observed in caspase-3 staining between groups $C$ and $Z X$, between groups $S$ and $X$, and between groups $\mathrm{S}$ and $\mathrm{ZX}(P=0.025, P=$ $0.021, P=0.009$, respectively) (Table 1, Figure 3).

The TEM findings and images revealed similar collagen fibril organization and undulation in groups $\mathrm{C}$ and $\mathrm{S}$. The fibrils were compact and randomly distributed in group $Z$, oriented in a parallel pattern in group $X$, more compact and tightly arranged in group ZX (Figure 4).

The collagen fibril diameters were higher in the non-interventional groups (groups $\mathrm{C}$ and $\mathrm{S}$ ). In the interventional groups, the collagen fibrils were thicker in group $Z$ than in the other groups (Tables 2 and 3). The distance between collagen fibrils was lowest in group $Z X$ and highest in group $X$ (Tables 2 and 3 ).

\section{DISCUSSION}

Oxygen radicals are present in normal tissues and cells. However, their effect is dose-dependent with a possible destructive effect on the cell wall structure and genetic material. ${ }^{[18]}$ Ozone $\left(\mathrm{O}_{3}\right)$ is normally found in the stratosphere and is used for conventional drinking water production at a concentration of $1-3 \mu \mathrm{l} / \mathrm{mL} .^{[19]}$ It is a highly reactive, unstable gas that spontaneously degrades to oxygen radicals and acts as an oxygen provider. ${ }^{[17]}$ There are three main types of molecular ozone reactions: (1) Electron transfer reactions, resulting in free oxygen radicals; (2) Oxygen-atom transfer reactions mainly occurring in inorganic materials; (3) Ozone addition reactions, which are the primary reactions occurring in organic compounds, resulting in double bond formation. ${ }^{[20]}$ Our study revealed that oxygen levels in the corneal stroma were higher in ozonated groups, which supports our primary goal, the oxygen-providing effect.

Several studies have evaluated the effects of ozone molecules on the eye. An experimental study revealed that ozone resulted in a decrease in goblet cell density and an increase in inflammatory cytokines on the ocular surface. ${ }^{[21]}$ Our study showed that corneal thickness was decreased in the ozonated groups (groups $Z$ and $Z X$ ). This 
Table 1. Between and within groups comparison of the examined parameters.

\begin{tabular}{|c|c|c|c|c|c|c|}
\hline & Group C & Group S & Group Z & Group X & Group ZX & $\mathbf{p}^{a}$ \\
\hline $\begin{array}{l}\text { Caspase activity, \%, } \\
\text { median (range) }\end{array}$ & $2.5(1-4)$ & $2(1-3)$ & $2.5(1-5)$ & $3(3-5)$ & $4(3-6)$ & $0.028^{*}$ \\
\hline $\begin{array}{l}\text { Oxygen saturation, \%, } \\
\text { median (range) }\end{array}$ & $18.7(14.5-22.2)$ & 16.7 (11.9-22.3) & $21.7(20.4-26.0)$ & $16.4(16.0-18.7)$ & $22.2(21.3-23.3)$ & $0.006^{* *}$ \\
\hline $\begin{array}{l}\mathrm{CCT}, \text { preop, mcm, } \\
\text { median (range) }\end{array}$ & $673.5(664-768)$ & 587.5 (545-639) & 637 (528-653) & $609(571-655)$ & 609 (545-652) & $0.006^{* * *}$ \\
\hline $\begin{array}{l}\text { CCT, postop, mcm, } \\
\text { median (range) }\end{array}$ & 673.5 (664-768) & 587.5 (545-639) & 569 (506-635) & $622(555-657)$ & $541(532-558)$ & $0.001^{* * * *}$ \\
\hline $\begin{array}{l}\text { Demarcation, mcm, } \\
\text { median (range) }\end{array}$ & & & 203.5 (185-257) & $190(185-245)$ & 323 (150-360) & 0.343 \\
\hline
\end{tabular}

Group C: control, Group S: sham, Group Z: ozonated only, Group X: cross-linked only, Group ZX: ozonated and crossl-linked, CCT: central corneal thickness, a: Kruskal-Wallis test

Between groups comparisons:

${ }^{*}$ Group C vs ZX, $p=0.0025$; Group S vs X, $p=0.021$; Group S vs ZX, $p=0.009$; Mann-Whitney U-test for each

${ }^{* *}$ Group C vs Z, $p=0.037$; Group C vs ZX, $p=0.018$; Group S vs Z, $p=0.025$; Group S vs ZX, $p=0.028$; Group Z vs X, $p=0.006$; Group X vs ZX, $p=0.009$, Mann-Whitney U-test for each

${ }^{* * *}$ Group C vs S, $p=0.004$; Group C vs Z, $p=0.004$; Group C vs X, $p=0.006$; Group C vs ZX, $p=0.006$; Mann-Whitney U-test for each

${ }^{* * * *}$ Group C vs S, $p=0.004$; Group C vs Z, $p=0.004$; Group C vs X, $p=0.006$; Group C vs ZX, $p=0.006$; Group S vs ZX, $p=$ 0.018; Group X vs ZX, $p=0.028$; Mann-Whitney U-test for each

Pre- vs post-operative pachymetry (CCT) comparison within study groups: Group Z: wilcoxon signed rank test, $p=0.028$;

Group X: wilcoxon signed rank test, $p=0.768$; Group ZX: wilcoxon signed rank test, $p=0.043$

Table 2. Collagen fibril diameter and distance between fibrils regarding the groups which were measured by TEM.

\begin{tabular}{lcc}
\hline Groups & Collagen diameter in nm, median (range) & $\begin{array}{c}\text { Distance between collagens in } \\
\text { (range) }\end{array}$ \\
\hline N (No intervention $[C+S])$ & $24,6(14.1-49.4)$ & $25.2(9.1-61.6)$ \\
Z & $23.7(16.7-35.0)$ & $24.3(11.5-44.6)$ \\
$X$ & $23.1(15.6-34.9)$ & $27.7(16.2-50.4)$ \\
ZX & $23.2(16.4-32.3)$ & $18.3(9.1-40.9)$
\end{tabular}

TEM, transmission electron microscope; N, no intervention (C: control and S: sham); Group C, control; Group S, sham; Group Z, ozonated only; Group X, cross-linked only; Group ZX, ozonated and cross-linked

Table 3. Between groups comparison of collagen fibril diameter and distance between fibrils.

\begin{tabular}{lcc}
\hline & Collagen fibril diameter (p-value $)^{a}$ & ${\text { Distance between collagen fibrils }(\mathrm{p} \text {-value })^{a}}$ \\
\hline Group N vs Z & $<0.001$ & 0.289 \\
Group N vs X & $<0.001$ & $<0.001$ \\
Group N vs ZX & $<0.001$ & $<0.001$ \\
Group Z vs X & 0.029 & $<0.001$ \\
Group Z vs ZX & 0.200 & $<0.001$ \\
Group X vs ZX & 0.337 & $<0.001$
\end{tabular}

N, no intervention (control and sham); Group Z, ozonated only; Group X, cross-linked only; Group ZX, ozonated and cross-linked; a, Mann-Whitney U-test 


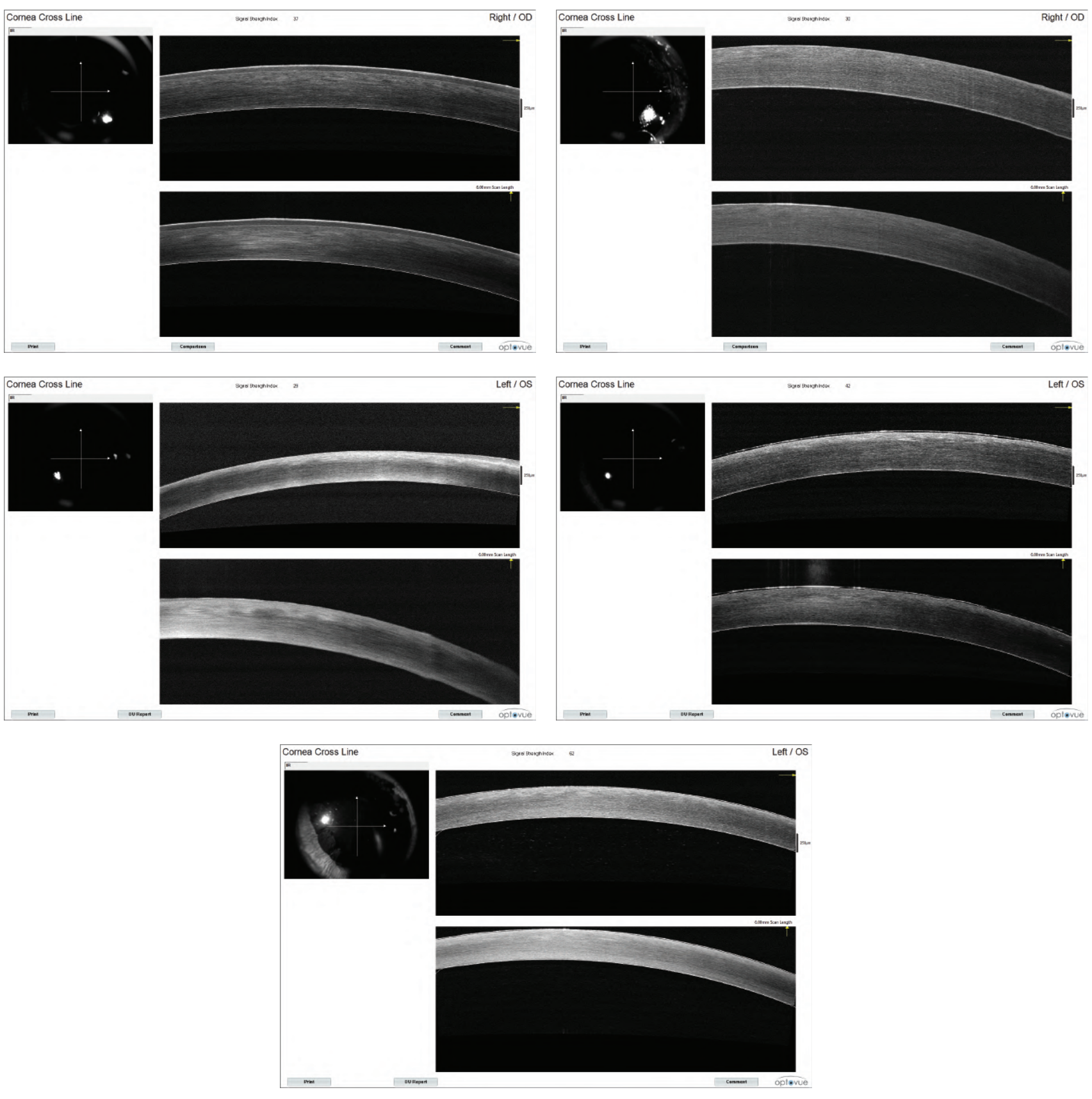

Figure 1. Optical Coherence Tomography corneal images of the groups control (a), sham (b), ozone (c), cross-linking (d), ozonecross-linking (e), respectively. Hyperreflectivity is increased in anterior stroma in groups $X$ and $Z$, but generalized in ZX.

observation can be explained by the use of liquid ozone which induced this decrease in thickness owing to its osmotic effect. To minimize this effect, we preferred the maximum possible concentration and the lowest exposure time. Wu et al ${ }^{[22]}$ have shown that ozone decomposes rapidly to diatomic oxygen and has a short half-life of 1-10 min in water. ${ }^{[22]}$ We applied ozone through a silica tube with an $8 \mathrm{~mm}$ aperture to limit the contact time within this range and prevent corneal edema.
Kamaew et al have shown that the stromal oxygen concentrations decrease within the first $15 \mathrm{sec}$ of UVA application and then rise to normal levels 3 min after cessation of UVA. ${ }^{[12]}$ Oxygen is necessary for the photochemical reaction to occur during the $\mathrm{CXL}$ procedure. ${ }^{[6]}$ In our study, the microprobe measurements showed higher corneal stromal oxygen levels in ozonated groups (groups Z and ZX), suggesting penetration of the topically applied ozone to the 

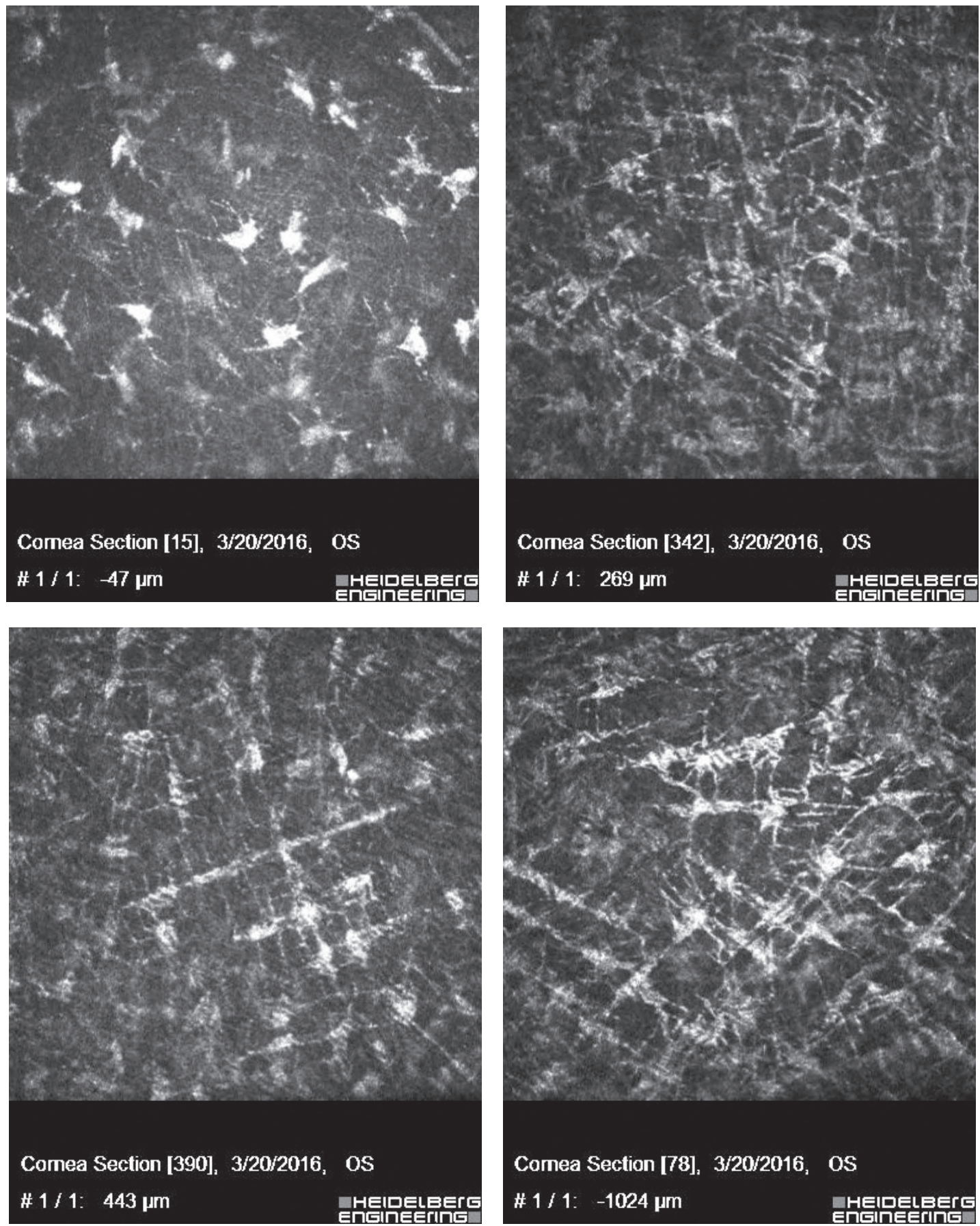

Figure 2. Corneal confocal images of groups sham (a), ozone (b), cross-linking (c), ozone-cross-linking (d), respectively. The keratocytes in stromal level shows straight extensions, which are signs of activity in all groups except control, which are exaggerated in ZX group.

stroma. This assumption, however, needs further refinement.

The standard CXL procedures lasts up to 60 min and a pulsed accelerated protocol has been developed to shorten the treatment duration without losing the therapeutic efficacy. ${ }^{[11,23]}$
Therefore, we aimed to augment the CXL effect in the pulsed accelerated protocol and, in the case of encouraging findings, implement this modification to clinical settings.

Early demarcation was previously described in an experimental study by Zhu et al; they postulated 

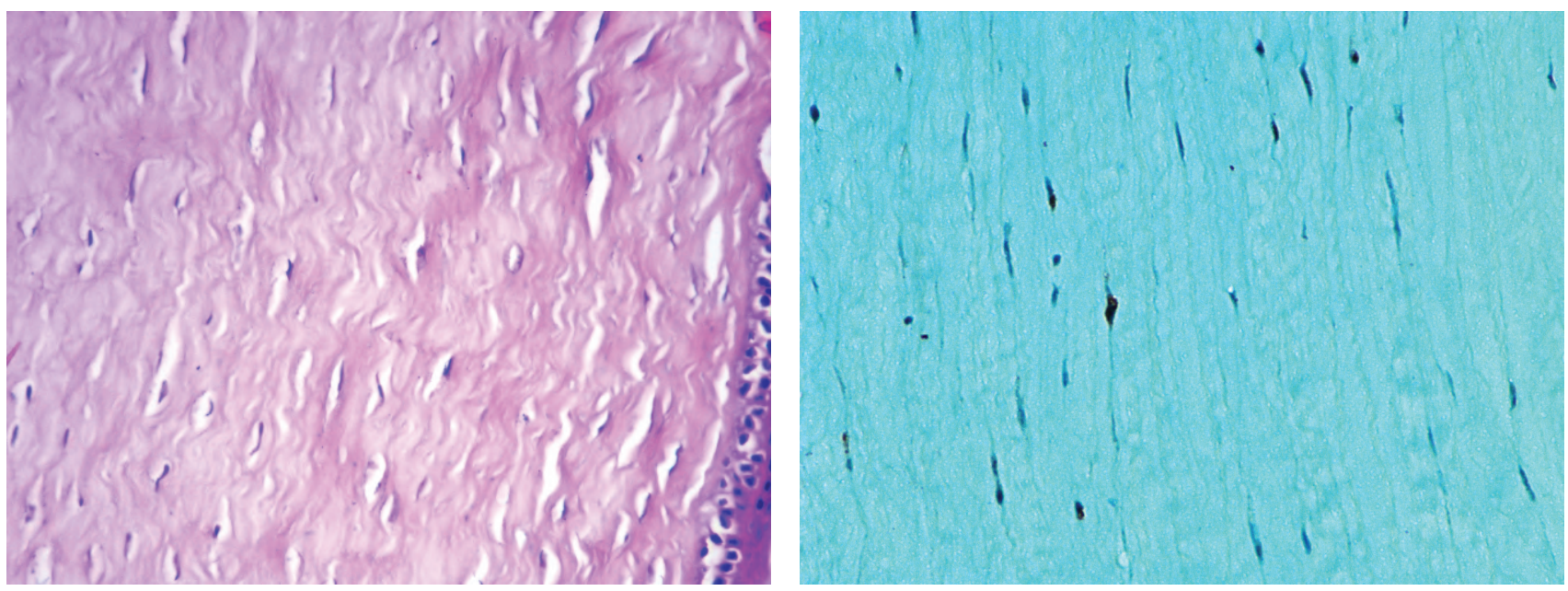

Figure 3. (a) A section of cornea from control group (HE X 400). (b) Positively stained nuclei in a section from ZX group (Caspase $\times 400)$.
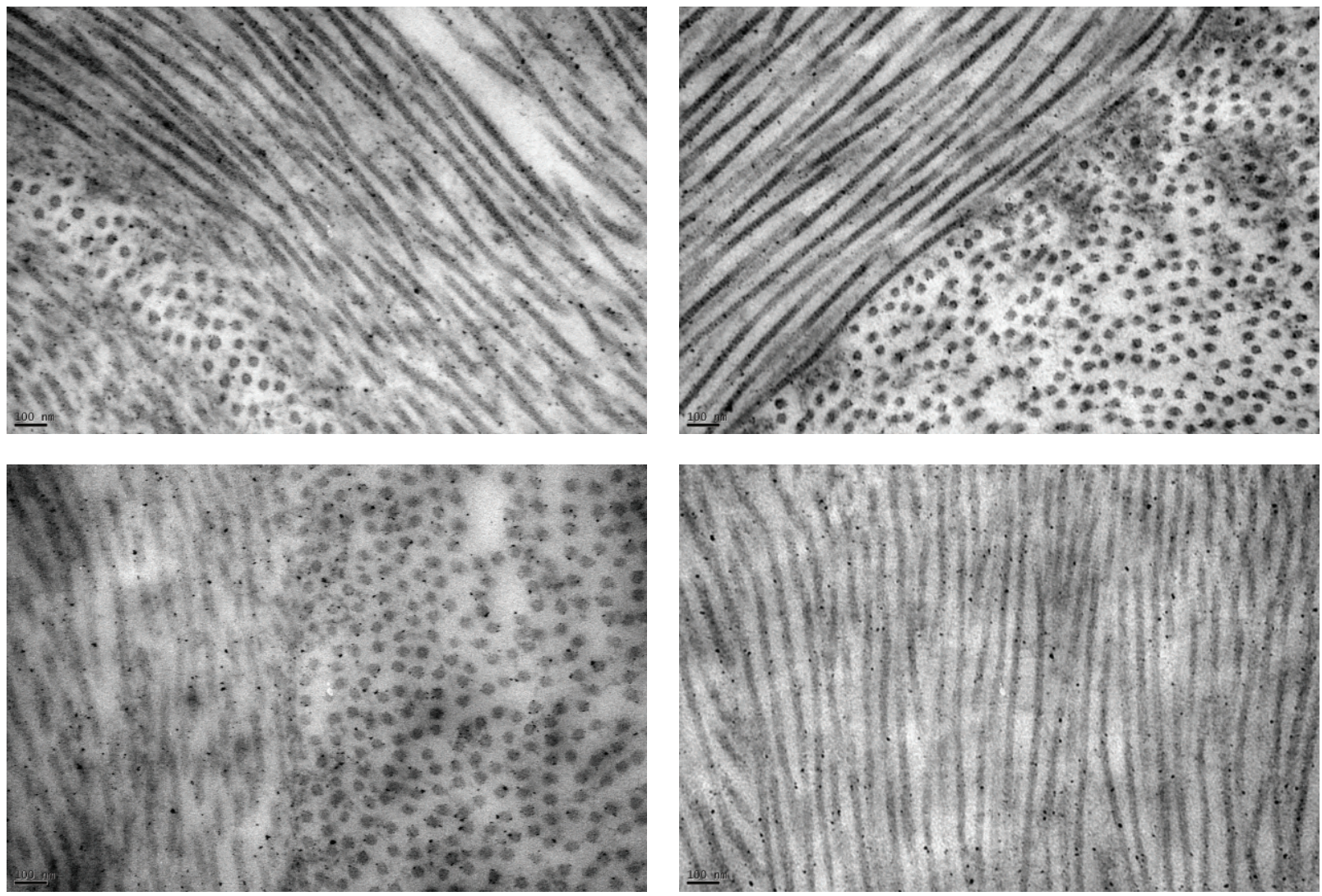

Figure 4. Images taken from transmissional electron microscopy of the corneas from groups (x100K); control (a), ozone (b), crosslinking (c), ozone-cross-linking (d), respectively. Collagen fibers and interlamellar spaces were examined by electron microscopy.

that the early apoptosis of keratocytes could induce increased light scattering and demarcation between the affected and unaffected stroma. ${ }^{[24]}$ We detected that demarcation lines in groups $Z$ and $Z X$ were similar to those in group $X$, indicating that the ozone itself triggers a chemical reaction similar to that observed in CXL. Post-interventional CCM findings demonstrated that increased stromal reflectivity and matrix striation were prominent in the anterior part in groups $\mathrm{Z}$ and $\mathrm{X}$, whereas these 
changes were present throughout the stromal layer in group ZX. These results confirmed that ozone can potentially augment the cross-linking effect of the classical CXL procedure.

Another study has indicated anterior stromal keratocyte apoptosis following CXL. ${ }^{[25]}$ We detected apoptosis with a higher caspase activity in cross-linking groups (groups $X$ and ZX), which demonstrates the efficacy of CXL.

$\mathrm{CXL}$ allows the reorganization of the corneal lamellar tissue by modifying the stromal collagen diameter and distance between collagen fibrils and proteoglycans. ${ }^{[26]}$ Clinical studies have revealed a mean collagen diameter of $30.8 \mathrm{~nm}$ and $32.2 \mathrm{~nm}$ in individuals younger and older than 65 years, respectively. ${ }^{[27]}$ It reveals that a natural cross-linking occurs non-enzymatically via free radicals with increasing age. ${ }^{[28]}$

Furthermore, we examined the effect of these procedures on collagen fibrils by utilizing TEM. The obtained images supported our hypothesis regarding the distribution pattern of collagen fibrils. In the ozonated group (group Z), the fibrils were denser than the controls and demonstrated a random distribution pattern. In the CXL group (group $X$ ), the fibrils were markedly condensed and presented an undulated pattern; in the $\mathrm{ZX}$ group, the undulation was less prominent and the distribution was more uniform and dense. These findings showed that the ozone application had an augmenting effect on the CXL procedure. Conversely, our measurements of fibril diameter and interfibrillar distance were not in accordance with our hypothesis. The fibril diameters of the study groups were similar but smaller than those of the control and sham groups, and interfibrillar distance was decreased from group $X$ to group $Z$ to group $Z X$ in descending order (Table 2 ). The small number of eyes in the groups, the random selection of four ultrastructural counting areas, and manual counting could be the principal explanations for this contradicting finding. ${ }^{[29]}$

Ozone is an inert, cheap, and easily available molecule that induces covalent chemical bonds resulting in cross-linking. Our study is the first to evaluate the effects of this molecule on the results of the CXL procedure. Our findings in cadaveric eyes can be regarded as preliminary to potential clinical applications. However, further animal studies are warranted to determine the most appropriate concentration, dosage, and duration of application of ozone as well as safety issues. These studies should evaluate the osmotic effects of the gaseous ozone form and the biomechanical properties of the treated corneas.

\section{Financial Support and Sponsorship}

This work was funded by the Scientific and Technological Research Council of Turkey (TUBITAK) [grant numbers: 115S862].

\section{Conflicts of Interest}

There are no conflicts of interest.

\section{REFERENCES}

1. Chen S, Mienaltowski MJ, Birk DE. Regulation of corneal stroma extracellular matrix assembly. Exp Eye Res 2015;133:69-80.

2. Gomes JA, Tan D, Rapuano CJ, Belin MW, Ambrósio R Jr, Guell JL, et al. Group of panelists for the Global Delphi Panel of Keratoconus and Ectatic Diseases. Global consensus on keratoconus and ectatic diseases. Cornea 2015;34:359-369.

3. Meek KM, Tuft SJ, Huang YF, Gill PS, Hayes S, Newton $\mathrm{RH}$, Bron AJ. Changes in collagen orientation and distribution in keratoconus corneas. Invest Ophthalmol Vis Sci 2005;46:1948-1956.

4. Wollensak G, Spoerl E, Seiler T. Riboflavin/ultraviolet a-induced collagen crosslinking for the treatment of keratoconus. Am J Ophthalmol 2003;135:620-627.

5. Wollensak G, Wilsch M, Spoerl E, Seiler T. Collagen fiber diameter in the rabbit cornea after collagen crosslinking by riboflavin/UVA. Cornea 2004;23:503-507.

6. Richoz O, Hammer A, Tabibian D, Gatzioufas Z, Hafezi F. The biomechanical effect of Corneal Collagen CrossLinking (CXL) with riboflavin and UV-A is oxygen dependent. Trans/ Vis Sci Technol 2013;2:6.

7. Filippello M, Stagni E, O'Brart D. Transepithelial corneal collagen crosslinking: bilateral study. J Cataract Refract Surg 2012;38:283-291.

8. Bikbova G, Bikbov M. Transepithelial corneal collagen cross-linking by iontophoresis of riboflavin. Acta Ophthalmol 2014;92:e30-e34.

9. Gu SF, Fan ZS, Wang LH, Tao XC, Zhang Y, Wang CQ, et al. A short-term study of corneal collagen cross-linking with hypo-osmolar riboflavin solution in keratoconic corneas. Int J Ophthalmol 2015;8:94-97.

10. Wernli J, Schumacher S, Spoerl E, Mrochen M. The efficacy of corneal cross-linking shows a sudden decrease with very high intensity UV light and short treatment time. Invest Ophthalmol Vis Sci 2013;54:1176-1180.

11. Mazzotta C, Traversi C, Caragiuli S, Rechichi M. Pulsed vs continuous light accelerated corneal collagen crosslinking: in vivo qualitative investigation by confocal microscopy and corneal OCT. Eye 2014;28:1179-1183. 
12. Kamaev P, Friedman MD, Sherr E, Muller D. Photochemical kinetics of corneal cross-linking with riboflavin. Invest Ophthalmol Vis Sci 2012;53:2360-2367.

13. Takemoto LJ, Hansen JS. Covalent and noncovalent interactions of membrane proteins from the chick lens. Exp Eye Res 1981;33:267-276.

14. Goosey JD, Zigler JS Jr, Kinoshita JH. Cross-linking of lens crystallins in a photodynamic system: a process mediated by singlet oxygen. Science 1980;208:1278-1280.

15. Fujimori E. Crosslinking and photoreaction of ozoneoxidized calf-lens alpha-crystallin. Invest Ophthalmol Vis Sci 1982;22:402-405.

16. Jernigan HM Jr. Role of hydrogen peroxide in riboflavinsensitized photodynamic damage to cultured rat lenses. Exp Eye Res 1985;41:121-129.

17. Pryor WA, Squadrito GL, Friedman M. The cascade mechanism to explain ozone toxicity: the role of lipid ozonation products. Free Radic Biol Med 1995;19:935-941.

18. Sanhueza PA, Reed GD, Davis WT, Miller TL. An environmental decision-making tool for evaluating ground-level ozone-related health effects. J Air Waste Manag Assoc 2003;53:1448-1459.

19. Hoigne J. Chemistry of aqueous ozone and transformation of pollutants by ozonation and advanced oxidation processes. In: Hutzinger $O$, editor. Quality and treatment of drinking water 2. The handbook of environmental chemistry. Volume 5 Water Pollution. Berlin: SpringerVerlag; 1998:83-143.

20. Von Gunten U. Ozonation of drinking water: part I. Oxidation kinetics and product formation. Water Res 2003;37:1443-1467.

21. Lee H, Kim EK, Kang SW, Kim JH, Hwang HJ, Kim TI. Effects of ozone exposure on the ocular surface. Free Radic Biol Med 2013;63:78-89.
22. Wu J, Doan H, Cuenca MA. Investigation of gaseous ozone as an anti-fungal fumigant for stored wheat. J Chem Technol Biotechnol 2006;81:1288-1293.

23. Vounotrypidis E, Athanasiou A, Kortüm K, Kook D, Shajari $\mathrm{M}$, Priglinger $\mathrm{S}$, et al. Long-term database analysis of conventional and accelerated crosslinked keratoconic mid-European eyes. Graefes Arch Clin Exp Ophthalmol 2018;256:1165-1172.

24. Zhu AY, Vianna LM, Borkenstein EM, Elisseeff J, Jun AS. Assessment of a novel corneal-shaping device with simultaneous corneal collagen cross-linking using a porcine eye model. Cornea 2016;35:114-121.

25. Messmer EM, Meyer P, Herwig MC, Loeffler KU, Schirra F, Seitz B, Thiel M, et al. Morphological and immunohistochemical changes after corneal cross-linking. Cornea 2013;32:111-117.

26. Akhtar S, Almubrad T, Paladini I, Mencucci R. Keratoconus corneal architecture after riboflavin/ultraviolet A crosslinking: ultrastructural studies. Mo/ Vis 2013;19:1526-1537.

27. Daxer A, Misof K, Grabner B, Ettl A, Fratzl P. Collagen fibrils in the human corneal stroma: structure and aging. Invest Ophthalmol Vis Sci 1998;39:644-648.

28. Malik NS, Moss SJ, Ahmed N, Furth AJ, Wall RS, Meek KM. Ageing of the human corneal stroma: structural and biochemical changes. Biochim Biophys Acta 1992;1138:222-228.

29. Quantock AJ, Winkler M, Parfitt GJ, Young RD, Brown DJ, Boote $C$, et al. From nano to macro: studying the hierarchical structure of the corneal extracellular matrix. Exp Eye Res 2015;133:81-99. 